\title{
Should Infliximab be Used as an Adjuvant to IVIG in the Treatment of Children With Kawasaki Disease Who Are at High Risk for Resistance to Conventional Therapy?
}

\author{
Shelby Davies • Gabrielle Gold-von Simson
}

Received: 10 May 2013/Accepted: 30 May 2013/Published online: 15 June 2013

(C) Springer Science+Business Media New York 2013

\section{To the Editor,}

The recently published article, entitled "Extensive Coronary Aneurysms with Thrombosis in Resistant Kawasaki Disease" by Sivakumar and Pavithran [4], concisely delineates the limitations in the treatment of Kawasaki disease (KD). There is no one accepted treatment algorithm for treatment-refractory patients, and the efficacy of infliximab as a first-line therapy adjunct has not yet been studied. Although a retrospective study showed improvement in various clinical outcomes, use of infliximab as second-line therapy is not convincingly supported by highly powered studies [5]. As this case illustrates, the treatment of $\mathrm{KD}$ is neither universal nor intuitive. The efficacy of infliximab in this population would perhaps be enhanced if administered at an earlier time point, especially in children who are at high risk for resistance to therapy.

Children who are resistant to first-line treatment with intravenous immunoglobulin (IVIG) carry a higher risk for development of coronary artery aneurysms [3]. Therefore, to avoid such devastating outcomes, should infliximab be used as an adjunct to IVIG in the treatment of children with $\mathrm{KD}$ who are at high risk? These high-risk patients (male, young age, recurrence of fever) could possibly be identified with a risk stratification algorithm and may benefit from more aggressive early therapy.

It has been shown that tumor-necrosis factor (TNF)-alpha inhibitors decrease inflammation and endoarteritis in murine models, specifically the inhibition of neutrophil adhesion to endothelial cells, which is seen in the early phase of vasculitis [2]. This proof-of-concept experiment further elucidates

S. Davies $(\bowtie) \cdot$ G. Gold-von Simson

New York University School of Medicine, 550 First Avenue, New York, NY 10016, USA

e-mail: shelby.davies@nyumc.org the role of TNF-alpha inhibitors in the treatment of panvasculitis. Based on the mechanism of endoarteritis and the success of other patients treated with infliximab, we believe that high-risk KD patients may benefit from early treatment with infliximab in conjunction with conventional IVIG therapy [1,3]. In keeping with this hypothesis, researchers at The University of California at San Diego recently completed a phase III placebo-controlled, multicenter, randomized clinical trial of infliximab plus standard therapy versus placebo plus standard therapy in children with acute KD to determine if the addition of infliximab to primary therapy can decrease the percentage of children who are resistant to therapy. Results of this study will perhaps further guide our primary management of KD.

\section{References}

1. Mori M, Imagawa T, Hara R, Kikuchi M, Hara T, Nozawa T et al (2012) Efficacy and limitation of infliximab treatment for children with Kawasaki disease intractable to intravenous immunoglobulin therapy: report of an open-label case series. J Rheumatol 39(4):864-867

2. Oharaseki T, Yokouchi Y, Yamada H, Mamada H, Muto S, Sadamoto K, et al. (2013) The role of TNF-alpha in a murine model of Kawasaki disease arteritis induced with a Candida albicans cell wall polysaccharide. Mod Rheumatol. doi:10.1007/ s10165-013-0865-4

3. Shirley DA, Stephens I (2010) Primary treatment of incomplete Kawasaki disease with infliximab and methylprednisolone in a patient with a contraindication to intravenous immunoglobulin. Pediatr Infect Dis J 29(10):978-979

4. Sivakumar K, Pavithran S (2013) Extensive coronary aneurysms with thrombosis in resistant Kawasaki disease. Pediatr Cardiol 34:444-446

5. Son MB, Gauvreau K, Burns JC, Corinaldesi E, Tremoulet AH, Watson VE et al (2011) Infliximab for intravenous immunoglobulin resistance in Kawasaki disease: a retrospective study. J Pediatr 158(4):644-649 